\title{
Study on Network Behavior Assessment Using Amazon Web and Cloud Computing Services
}

\author{
Lamyaa Mohammed Kadhim \\ College of Dentistry \\ University of Al-Mustansiriyah \\ Baghdad, Iraq
}

\begin{abstract}
The revolution of Cloud Computing increases the opportunities to provide realistic and most sophisticated evaluation modules that reduce the management time and cost of network performance evaluation and failure prediction. In our research, This paper presents a cloud-based software system that utilizing the Amazon Elastic MapReducer (EMR) ensemble clustered instances for evaluating the collected network measurements to quantifies network performance and predicate its degradation in the long run. The extracted outcomes illustrate the efficiency of the proposed system.
\end{abstract}

Keywords: Amazon Web Services, Network Failure, Amazon, Elastic Map Reducer.

\section{INTRODUCTION}

The network monitoring is the most critical aspect in the network management the tech companies in order to provide a better reliable system for their customers. However, the network reliability is the most significant challenge to maintain the network consistently performs according to its specifications.

Network failure can be referred to any network device or component may halt or work inefficiently under wide range of possible conditions. In order to analyze the network behavior, huge amount of data need to be collected, tested, and studied to come up with clear view of how the network failure can be predicted. In this research, two different Python programs have been developed to collect and examine sequence of network patterns and calculate some statistics of abnormal patterns using AWS.

In our previous work, Joint Clustering and Association Analysis approach (JCAA) has been developed to predict the network degradation by classifying the collected data into groups of k-means clusters and discover the relationships between groups using association analysis. The proposed approach demonstrated the ability to improve the quality of predication by discovering the root cause of network failure and proactively correct the network drawbacks in a timely manner. [1].

We need to emphasize here; the proposed work is an extension of the JCAA in term of overcoming the machines low performance while processing massive network measurement and reduce processing time.

The following paragraphs are arranged according to the research policy being undertaken. The first section represents a brief review of the studies that have been accomplished in network performance degradation and failure predication. The second section gives an illustration of the principle core of AWS and the most related solution provided to support network traffic management. The third section presents the proposed approach for network failure evaluation using AWS. Testing and evaluating the constructed results from the proposed approach will be described in the section four. Through section five, the conclusion and suggestions for the future works will be described.

\section{AMAZON ELASTIC MAPREDUCER (EMR)}

Amazon offers efficient and proactive tools to process and analysis data across distributed cluster of virtual servers on Elastic Compute Cloud (EC2) and Amazon Simple Storage Service (S3). Amazon Elastic MapReducer (EMR) is one of sophisticated tools built on top of Apache Hadoop, a Java-based framework that supports large data storage in distributed environment, 
provided for big data processing. EMR is built on top of Apache Hadoop, a software platform that supports massive data sets storage in disseminated computing environment [2-3].

\subsection{THE PROPOSED SYSTEM}

The proposed system is devoted to provide a vital analytical tool to measure the network substrate performance and proactively react to network degradation. We tend to focus on analyzing massive network measurements by utilizing distributed environment. AWS is the best match as a highly customized network environment in support of concurrent instance.

We move our system from adapting local machines to distributed environment that allows utilizing concurrent instances to run the developed programs in real-time without The Proposed system reshapes from two parts:

\section{PYTHON LOCAL MACHINE PROGRAM}

This part of the developed system runs on single machine to collect the network patterns from virtual network. The following algorithm represents the main steps of this part.

1. Grouping the similar patterns

2. Averaging the grouped patterns to reduce the computation

3. Encoding the averaged patterns in a sequence of binary streams

4. Extracting the failure paths and find the failure average pattern.

\section{PYTHON PROGRAMS}

The Mappers and reducers program has been developed to collect network patterns and compute important network statistics, such averaging, average failure pattern, etc. The Amazon Elastic MapReduce service (EMR) has been utilized to deploy the programs which provides flexible distributed environment that support the MapReduce approach. The program runs through the following steps:

4.1. Access to the AWS (Amazon Web Service) using registered account

4.2. On Amazon S3, create a bucket and name it patterns to store the collected network measurements

4.3. Download project files from the following URL:

4.3.1 Network input patterns: http://pattern.s3.amazonaws.com/heatmap files/heatmaplabel1000.txt, and upload the file to bucket pattern/input

4.3.2 Averaging mapper : http://pattern.s3.amazonaws.com/map/pattmapper.py , and upload it to the bucket pattern/map

4.3.3 Averaging reducer : http://pattern.s3.amazonaws.com/reduce/pattreducer.py and upload it to the bucket pattern/reduce

4. 4. Go to Amazon Elastic MapReduce and do the following:

4.4.1 create New Job Flow

4.4.2 Specify the Job name and choose Run your Own application from Create a Job Flow option, and choose Streaming option. Then hit continue

4.4.3 Put all necessary information in fields like bellow:

Input location: pattern/input

Output location: pattern/output

Mapper : pattern/map/pattmapper.py

Reducer: pattern/reduce/pattreducer.py

And hit continue

4.5 Specify the number of Instance, by default you can choose 2 instances, and hit continue and continue.

4.6 Check the information and hit Create Job Flow. 
After the running job is finished, go to the pattern bucket and then to the output sub-folder, and download the output files. These files normally start with prefix part-xxxxx.

4.6- Download the merger.py file from the following URL and save it on the same directory of part-xxxxx files:

http://pattern.s3.amazonaws.com/localmachine/Merger.py

4.7- Go to the merger.py folder and run from the command line:

$$
\text { /> Python merger.py. }
$$

The following figures show the steps of how to create AWS MapReduce job:

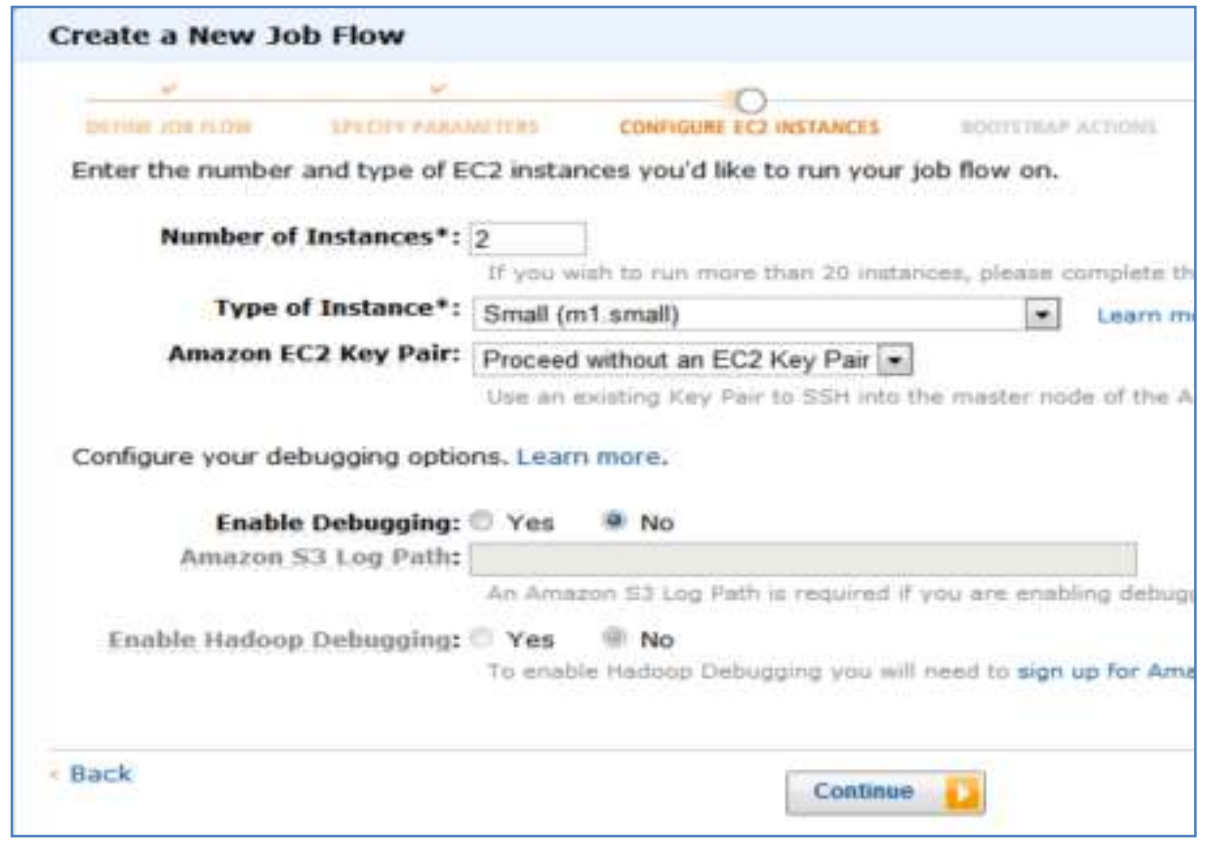

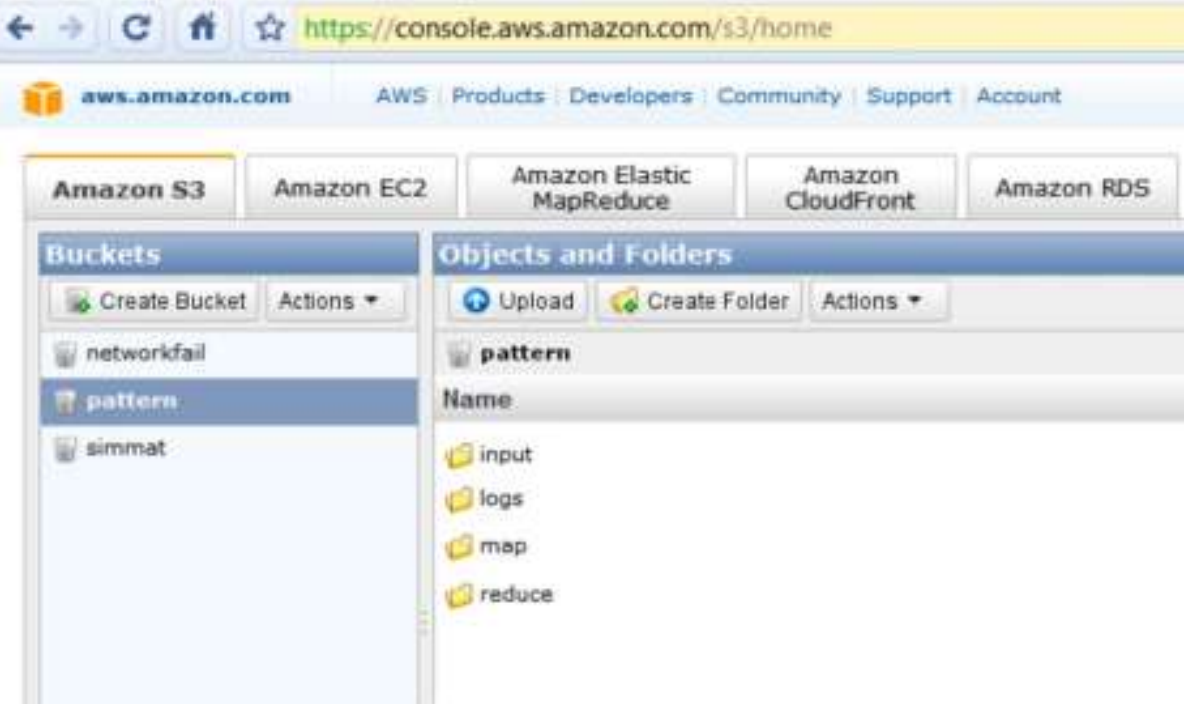




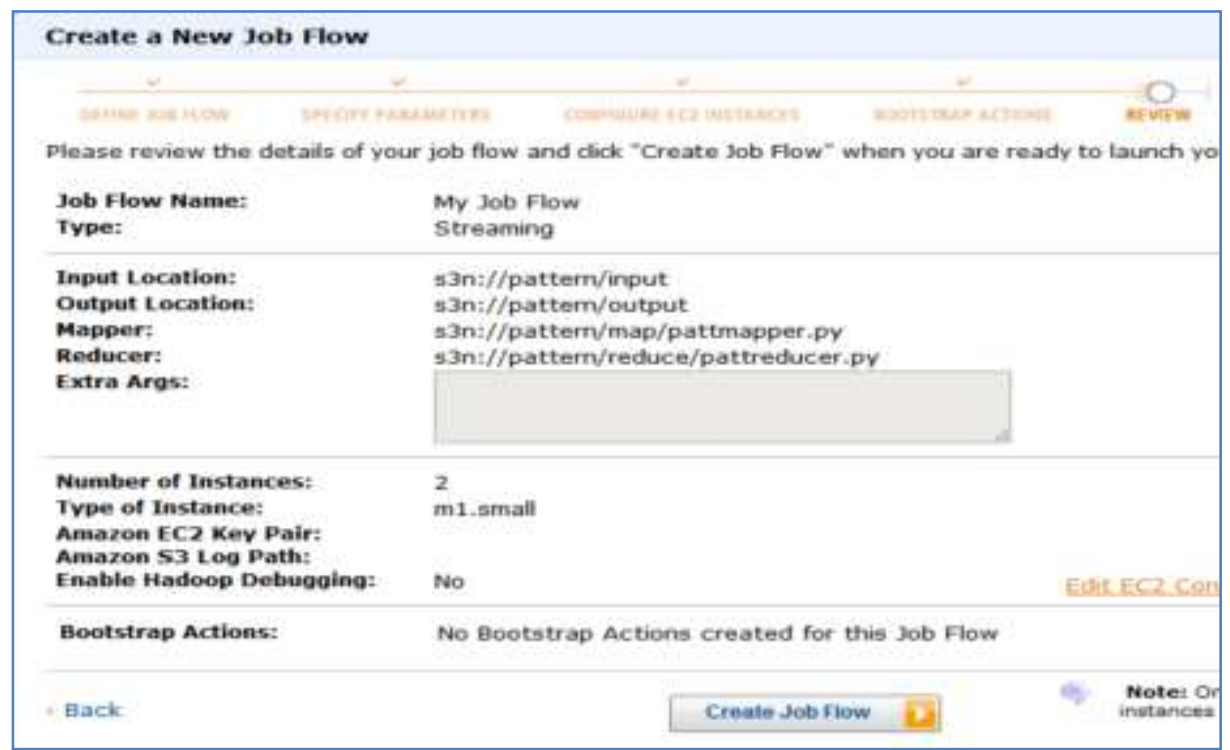

\section{Create a New Job Flow}

orrave joa now

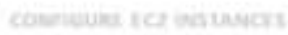

Magramen actio

Creating a job flow to process your data using Amazon Elastic MapReduce is simple an name and selecting its type. If you don't already have an application you'd like to run o available to help you get started.

available to help you get started.

Job Flow Name* : netpatterl

Job Flow Name doesn't need to be unidue. We supoest you give it:

Create a Job Flow*: o Run your own application

Run a sample application

A streaming job f of map and reduc: Amazon 53. The $f$ the supported lan Streaming Bash, $\mathrm{C}++$. 


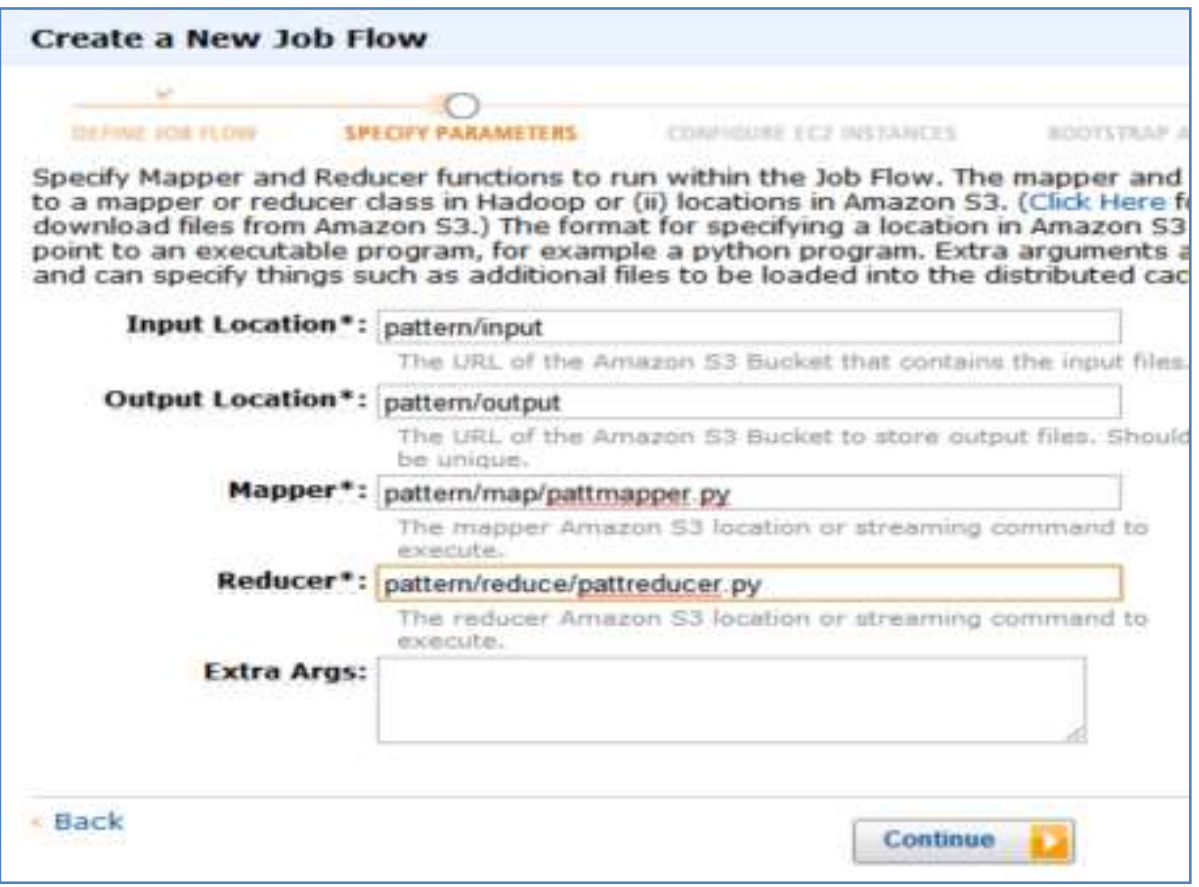

\section{EXPECTED OUTCOMES}

As we mentioned above, the primary goal of the present study is to discover risky network patterns from a network systems perspective. Towards this end, we will work at improving the techniques currently being used by both researchers and practitioners using Amazon Web Services. More precisely, the following are the key takeaway from the conducted study:

1- Designing and building a state-of-the-art Elastic MapReduce framework for optimizing computational resources utilization on network Systems,

2- The analysis and improvement of reinforcement prediction algorithm using a dedicated Amazon networks probe, and

3- Improve the computational performance of the proposed cloud-based system by populating the reinforcement algorithm through AWS techniques.

\section{REFERENCES}

[1] A. Al-Fuqaha, A. Rayes, D. Kountanis, H. Abed, A. Kamel, R. Salih, "Prediction of Performance Degradation in Telecommunication Networks Using Joint Clustering and Association Analysis Techniques," IEEE International Workshop on Management of Emerging Networks and Services, IEEE Globecom 2010, Miami, Florida, 6-10 Dec. 2010.

[2] Z. Liu, D. Um," Analysis of resource usage profile for MapReduce applications using Hadoop on the cloud", IEEE 2012 International Conference on Quality, Reliability, Risk, Maintenance, and Safety Engineering, Chengdu, China. 2012.

[3] Xueyuan, Brian, Yuansong, "Experimental evaluation of memory configurations of Hadoop in Docker environments", Signals and Systems Conference (ISSC) 2016 27th Irish, pp. 1-6, 2016.

[4] D. Dahiphale, R. Karve, A. Vasilakos, H. Liu, Z. Yu, A. Chhajer, J. Wang, and C. Wang, “An Advanced MapReduce: Cloud MapReduce, Enhancements and Applications”, IEEE Transactions On Network And Service Management, Vol. 11, No. 1, March 2014.

[5] Z. Liu and D. Mu, "Analysis of resource usage profile for MapReduce applications using Hadoop on cloud," 2012 International Conference on Quality, Reliability, Risk, Maintenance, and Safety Engineering, Chengdu, 2012, pp. 1500-1504.

[6] M. Adnan, M. Afzal, M. Aslam, R. Jan and A. M. Martinez-Enriquez, "Minimizing big data problems using cloud computing based on Hadoop architecture," 2014 11th Annual High Capacity Optical Networks and Emerging/Enabling Technologies (Photonics for Energy), Charlotte, NC, 2014, pp. 99-103.

[7] J. M. Luna, C. T. Abdallah and G. L. Heileman, "Probabilistic Optimization of Resource Distribution and Encryption for Data Storage in the Cloud," in IEEE Transactions on Cloud Computing, vol. 6, no. 2, pp. 428-439, 1 April-June 2018. 
[8] P. Pierleoni, R. Concetti, A. Belli and L. Palma, "Amazon, Google and Microsoft Solutions for IoT: Architectures and a Performance Comparison," in IEEE Access, vol. 8, $\quad$ pp. 2020. doi: 10.1109/ACCESS.2019.2961511

[9] A. J. Sanad and M. Hammad, "Reducing Cloud provisioning Cost Using Spot Instances hopping," 2019 International Conference on Innovation and Intelligence for Informatics, Computing, and Technologies (3ICT), Sakhier, Bahrain, 2019 , pp. 1-6.

[10] A. Chiniah, A. Chummun and Z. Burkutally, "Categorising AWS Common Crawl Dataset using MapReduce," 2019 Conference on Next Generation Computing Applications (NextComp), Mauritius, 2019, pp. 1-6. 